\title{
Quality of life of colorectal cancer survivors participating in a pilot randomized controlled trial of physical activity trackers and daily text messages
}

\author{
Hilary Chan ${ }^{1} \cdot$ Katherine Van Loon ${ }^{2,3} \cdot$ Stacey A. Kenfield ${ }^{4}$. June M. Chan ${ }^{4,5} \cdot$ Emily Mitchell $^{2} \cdot$ Li Zhang $^{2,3,5}$. \\ Alan Paciorek ${ }^{3,5}$. Galen Joseph ${ }^{6}$. Angela Laffan ${ }^{3}$. Chloe Atreya ${ }^{2,3}$. Yoshimi Fukuoka ${ }^{7}$. Christine Miaskowski . $^{7}$ \\ Jeffrey A. Meyerhardt ${ }^{8}$. Alan P. Venook ${ }^{2,3}$ • Erin L. Van Blarigan ${ }^{4,5}$
}

Received: 6 May 2021 / Accepted: 27 January 2022 / Published online: 4 February 2022

(c) The Author(s) 2022

\begin{abstract}
Purpose There are over 1.3 million colorectal cancer (CRC) survivors in the USA, many of whom report lower health-related quality of life (HRQoL) years after treatment. This study aimed to explore the effect of digital health tools on HRQoL in CRC survivors.

Methods We conducted a two-arm, randomized controlled trial of 42 subjects who had completed treatment for CRC. Participants in the intervention arm received a Fitbit Flex ${ }^{\mathrm{TM}}$ and daily text messages for 12 weeks. HRQoL was assessed as a secondary endpoint in both arms at enrollment and 12 weeks using the Medical Outcomes Study Short Form Survey (SF-36) and the Functional Assessment of Cancer Therapy-Colorectal (FACT-C). Survey score changes from enrollment to 12 weeks were compared between the two arms using independent $t$ tests, and scores at enrollment and 12 weeks were compared using paired $t$ tests.

Results An increase in the FACT-C functional well-being subscale was observed in individuals in the intervention arm pre- to post-intervention (median difference, 2 ; interquartile range (IQR), 1,$4 ; P=.02$ ). Although the between-group comparison was not statistically significant, no change in the functional well-being subscale was observed in the control arm (median difference, $0 ; \mathrm{IQR}, 1,1 ; P=.71$ ). No other measures of HRQoL appeared to differ within arm across time points or between arms.

Conclusion A 12-week digital physical activity intervention may improve functional well-being among CRC survivors. Larger randomized studies are needed to determine if digital health tools improve functional well-being among CRC survivors and if this improvement can be sustained over time.
\end{abstract}

Trial registration NCT02966054; registration date, November 17, 2016

Keywords Colorectal cancer · Quality of life

Erin L. Van Blarigan

erin.vanblarigan@ucsf.edu

1 Department of Medicine, University of California San Francisco, San Francisco, CA, USA

2 Division of Hematology/Oncology, Department of Medicine, University of California San Francisco, San Francisco, CA, USA

3 Helen Diller Family Comprehensive Cancer Center, University of California San Francisco, San Francisco, CA, USA

4 Department of Urology, University of California San Francisco, UCSF Box 0560, 16th Street, 2nd Floor, San Francisco, CA 94158, USA
5 Department of Epidemiology and Biostatistics, University of California San Francisco, San Francisco, CA, USA

6 Department of Anthropology, History, and Social Medicine, University of California San Francisco, San Francisco, CA, USA

7 Department of Physiological Nursing, University of California San Francisco, San Francisco, CA, USA

8 Dana-Farber/Partners CancerCare, Boston, MA, USA 


\section{Introduction}

Colorectal cancer (CRC) is the fourth most common cancer diagnosed in the USA [1]. As mortality from CRC decreases, the prevalence of survivors continues to rise, with an estimated 1.3 million CRC survivors in the USA in 2014 [1]. With the growing number of CRC survivors, health-related quality of life (HRQoL) becomes an increasingly important patient reported outcome.

CRC survivors often report lower HRQoL compared to the general population [2]. These differences, particularly in the physical functioning domain, are greatest early after treatment $[3,4]$. While fairly high overall HRQoL scores have been reported many years after treatment, deficits in physical, emotional, and social functioning may last for more than 3 years after treatment [5, 6]. Furthermore, some studies suggest that these deficits in HRQoL, specifically in younger CRC survivors, can persist for up to 10 years after diagnosis [7].

The effects of exercise on HRQoL have been well-studied in cancer survivors. A meta-analysis of 40 randomized controlled trials (RCTs), involving all types of exercise, suggested that physical activity in breast, colorectal, head and neck, lymphoma, and other cancer survivors resulted in significantly improved global HRQoL at 12 weeks and 6 months [8]. Similarly, in a recent review of 12 observational studies, physical activity was consistently associated with improved survival outcomes and better HRQoL in CRC survivors. [9] Furthermore, a cross-sectional study of 145 CRC survivors reported that sedentary behavior was associated with significantly reduced global quality of life and high disability [10].

These data come from observational studies with selfreported physical activity as well as randomized controlled trials with supervised exercise interventions. Few studies have examined digital health tools that promote physical activity (e.g., physical activity trackers and daily text messages) in relation to quality of life in CRC survivors. We hypothesized that such tools may be a low-cost intervention that improves physical activity and HRQoL in this population. Thus, we included HRQoL as a secondary endpoint in our pilot randomized controlled trial testing the feasibility and acceptability of a digital physical activity intervention in CRC survivors.

Smart Pace was a 1:1 pilot randomized controlled trial of 42 CRC survivors to assess the feasibility of using digital health tools (physical activity trackers and daily text messages). The primary outcome of feasibility was reached, as the intervention was found to be acceptable to CRC survivors [11]. Additionally, while not statistically significant, moderate-to-vigorous physical activity increased by $13 \mathrm{~min}$ more per day in the intervention arm compared to that in the control arm. An a priori secondary outcome of this pilot study was to explore the impact of the intervention on HRQoL to inform future studies. In this manuscript, we report on the change in HRQoL among CRC survivors participating in this pilot trial of a digital health intervention.

\section{Methods}

\section{Study population}

This study was a 1:1 pilot randomized controlled trial of 42 CRC survivors to assess the feasibility of digital health tools for promoting physical activity (NCT02966054) [11]. Potential study participants were identified using the University of California, San Francisco (UCSF) Cancer Registry, and through review of provider schedules in the gastrointestinal oncology practice at the UCSF Helen Diller Family Comprehensive Cancer Center. A detailed description of the eligibility criteria has been previously published [11]. Briefly, eligible survivors included those who had colon or rectal adenocarcinoma and were considered disease-free at enrollment. Eligibility criteria also included English reading and writing proficiency, Internet and mobile phone access, and the ability to navigate websites. Exclusion criteria included survivors who were very active at enrollment (defined as self-reported exercise for $\geq 30 \mathrm{~min}$ on $\geq 5$ days per week) and those with any medical contraindications to moderate-to-vigorous physical activity (MVPA).

A CONSORT flow diagram can be found in the first publication from this study and is included in Supplemental Fig. 1 [11]. We randomized 42 patients, 21 to the intervention arm and 21 to the control arm. One patient assigned to the intervention arm withdrew from the study prior to receiving the intervention materials after an incidental diagnosis of primary lung cancer, leaving 41 patients who contributed to this analysis of HRQoL. This study was reviewed and approved by the UCSF Institutional Review Board, and all patients provided informed consent prior to enrollment.

\section{Intervention and control arms}

Participants in the intervention arm received a Fitbit Flex ${ }^{\mathrm{TM}}$, daily text messages, and print education material. Participants in the control arm received print education material. The intervention content was based on the Theory of Planned Behavior [12]. The content of the text messages and education materials provided participants with informational and motivational messages on various exercise activities, such as brisk walking, jogging, and resistance training. Adherence to the intervention (Fitbit wear, text message responses) was high [11]. During the 12-week study period, participants in the intervention arm wore their Fitbit a median of 74 days 
( $88 \%$ of days in the study period; interquartile range (IQR), 23-83 days) [11].

\section{Quality of life assessments}

We measured colorectal cancer-specific quality of life using the Functional Assessment of Cancer Therapy-Colorectal (FACT-C) and general HRQoL using the Medical Outcomes Study Short Form-36 (SF-36) at enrollment and 12 weeks in all participants. Surveys were administered on the web using the UCSF Research Electronic Data Capture (REDCap) system [13]. The FACT-C questionnaire is comprised of 36 questions, 5 subscales, and 3 composite scores. The 5 subscales (physical well-being, social well-being, emotional well-being, functional well-being, and CRC subscale) are a scaled average of 6-7 individual questions $[14,15]$. The 3 composite scores (FACT-C total score, FACT-General (FACT-G), and FACT-Trial Outcome Index (FACT-TOI)) are sums of 3, 4, or 5 pertinent subscales. A higher score indicates better HRQoL.

The SF-36 version 1.0 is comprised of 36 questions, 9 domain scores, and 2 component summary scores [16]. The 9 domain scores (physical function, role physical, bodily pain, general health, vitality, social function, role emotional, mental health, and health transition) are comprised of the average of 1 to 10 questions. All 9 domain scores are weighted and combined to calculate both summary scores (physical health component and mental health component). Each summary score is weighted to an average value of 50 and standard deviation of 10 [17]. A higher score indicates better HRQoL. The question "Does your health now limit you in climbing one flight of stairs?" was omitted by mistake from the online REDCap surveys, so the value of that individual item was set to missing for all participants and time points. For the domain physical function (PF) that contained the question about the ability to climb one flight of stairs, we calculated the average score using the available 9 items. All domains and component summary scores were calculable.

\section{Physical activity tracking}

Physical activity was measured at enrollment and 12 weeks using Actigraph GTX3+ accelerometers. All participants were asked to wear the accelerometer on a belt around their waist for 7 consecutive days. Wear time was validated using Troiano 2007 settings in the ActiLife v6.13.3 software [18, 19]. We required at least $10 \mathrm{~h}$ of wear time per $24-\mathrm{h}$ period to define a valid day and a minimum of 3 valid days out of the 7 days that the participants were asked to wear the devices. Physical activity was categorized as sedentary (0-99 counts per minute), light (100-2019 counts per minute), moderate (2020-5998 counts per minute), and vigorous (5999 or more counts per minute) using Troiano 2008 cut-points [19]. Our primary measure of physical activity in the pilot study was MVPA, calculated as the sum of time spent performing moderate and vigorous activity.

\section{Statistical analysis}

Survey score changes from enrollment to 12 weeks were compared between the two arms using Wilcoxon signed rank tests, and scores at enrollment and 12 weeks were compared using Wilcoxon rank sum tests. Average effect of intervention on QOL change score was calculated using linear regression as score at 12 weeks minus score at enrollment [20]. SAS $®$ version 9.4 statistical computing software (Cary, North Carolina) was used for analysis, and statistical significance was declared at $p<0.05$. All analyses were intention-to-treat.

\section{Results}

No differences in age, body mass index (BMI), sex, race, education, employment status, marital status, or stage of diagnosis were found between the intervention and control arms at enrollment (Table 1). By chance, control participants appeared to exercise more at enrollment than the intervention arm participants (mean, $51 \mathrm{~min}$ per day compared to $33 \mathrm{~min}$ per day, respectively).

\section{Functional Assessment of Cancer Therapy- Colorectal}

Changes in FACT-C scores within and between treatment arms are summarized in Table 2. No differences were found in any of the FACT-C scores between the intervention and control arm at enrollment. Among participants in the intervention arm, the FACT-C score total score increased by a median difference of 5 (IQR, -5, 9) from enrollment to 12 weeks. In the intervention arm, there was an increase in the functional well-being subscale (median difference, 2; IQR, 1, 4) from enrollment to 12 weeks. In addition, in the intervention arm, there was an increase in the FACT-G score (median difference, 6; IQR, -2, 9) from enrollment to 12 weeks. In the control arm, no difference was observed in the functional well-being subscale between enrollment and 12 weeks (median difference, $0 ; \mathrm{IQR},-1,1$ ) nor was there a difference in the FACT-G score between enrollment and 12 weeks (median difference, 0 ; IQR, -8, 7). No other FACT$\mathrm{C}$ subscales, including the $\mathrm{CRC}$ subscale, appeared to differ across time points within arm or between arms.

\section{Short Form-36 Vitality Survey}

Changes in SF-36 scores within and between arms are summarized in Table 3. Scores at enrollment did not differ between the 
Table 1 Demographic characteristics, clinical factors, and physical activity at enrollment of 41 colorectal cancer survivors in a pilot randomized controlled trial of a digital health physical activity intervention

\begin{tabular}{|c|c|c|c|}
\hline Characteristic & Intervention $(n=20)$ & Control $(n=21)$ & $p$ value $^{\mathrm{a}}$ \\
\hline Age, years, mean $\pm \mathrm{SD}$ & $55.6(12.3)$ & $54.4(10.6)$ & 0.755 \\
\hline $\mathrm{BMI}, \mathrm{kg} / \mathrm{m}^{2}$, mean $\pm \mathrm{SD}$ & $29.7(7.2)$ & $27.1(4.3)$ & 0.162 \\
\hline Gender, $N(\%)$ & & & 0.852 \\
\hline Male & $8(40 \%)$ & $9(43 \%)$ & \\
\hline Female & $12(60 \%)$ & $12(57 \%)$ & \\
\hline Race, $N(\%)$ & & & 0.680 \\
\hline Asian & $2(10 \%)$ & $3(14 \%)$ & \\
\hline Black & $1(5 \%)$ & $0(0 \%)$ & \\
\hline Native American, Pacific Islander, or Other & $3(15 \%)$ & $2(10 \%)$ & \\
\hline White & $14(70 \%)$ & $16(76 \%)$ & \\
\hline College degree, $N(\%)$ & $17(85 \%)$ & $21(100 \%)$ & 0.065 \\
\hline Works full-time, $N(\%)$ & $14(70 \%)$ & $12(57 \%)$ & 0.392 \\
\hline Married, $N(\%)$ & $9(45 \%)$ & $11(52 \%)$ & 0.636 \\
\hline Cancer, $N(\%)$ & & & 0.890 \\
\hline Colon cancer & $11(55 \%)$ & $12(57 \%)$ & \\
\hline Rectal cancer & $9(45 \%)$ & $9(43 \%)$ & \\
\hline Years since diagnosis, median [range] & $1[0,8]$ & $1[0,4]$ & 0.526 \\
\hline Tumor stage, $N(\%)$ & & & 0.369 \\
\hline I & $4(20 \%)$ & $4(19 \%)$ & \\
\hline II & $2(10 \%)$ & $6(29 \%)$ & \\
\hline III & $13(65 \%)$ & $11(52 \%)$ & \\
\hline IV & $1(5 \%)$ & 0 & \\
\hline $\begin{array}{l}\text { Moderate-to-vigorous physical activity (minutes/ } \\
\text { day), mean } \pm \mathrm{SD}^{\mathrm{b}}\end{array}$ & $32.9(17.9)$ & $50.8(20.7)$ & 0.005 \\
\hline Steps per day, mean $\pm \mathrm{SD}^{\mathrm{b}}$ & 9008 (3639) & $11830(4052)$ & 0.024 \\
\hline
\end{tabular}

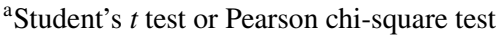

${ }^{\mathrm{b}}$ Average daily moderate-to-vigorous physical activity and steps were measured using 7 days of Actigraph GT3X+ accelerometers

intervention arm and the control arm. There were no differences in the physical or mental health component summary scores from enrollment to 12 weeks within or between the intervention or control arms. While the median change in the physical subscore was 0 for both arms, there was a wide range in changes from 0 to 12 weeks for this measure (controls median difference, 0; IQR, 0, 38; intervention median difference, 0; IQR, 0 , 33). In addition, there was a suggestion of an increase in the vitality sub-score, a measure of energy and fatigue, observed in the intervention arm from enrollment to 12 weeks (median difference, 10; IQR, 0, 20). No other SF-36 sub-scores differed across time points within arms or between arms.

\section{Discussion}

In this pilot RCT, we observed little change in overall HRQoL among CRC survivors participating in 12 weeks of physical activity trackers and daily text messages or usual care. However, we did note small improvements in functional well-being among CRC survivors randomized to the intervention, with no change in this domain among controls.
There may be several reasons for the overall lack of change in HRQoL observed in our pilot study. One explanation is the relatively high rates of physical activity among our study population at enrollment. Almost $90 \%$ (36 out of 41) of our survivors met or exceeded the recommended amount of physical activity of 150 min per week for cancer survivors at enrollment based on the accelerometers but were still eligible for participation because they had reported on the screening survey that they engaged in $30 \mathrm{~min}$ or more of exercise on fewer than 5 days per week [21]. Thus, participants may already have achieved near maximum benefits in HRQoL from physical activity prior to enrollment, which would minimize any observed effect of the digital health intervention on HRQoL. This finding should be considered in determining eligibility criteria for future larger-scale intervention trials, with consideration for targeting individuals with lower levels of physical activity at baseline.

Relatedly, participants at enrollment in both arms had HRQoL scores similar to the general population. The mean SF-36 physical and mental health component summary scores at enrollment in our participants were within the mean and one standard deviation of normalized scores 


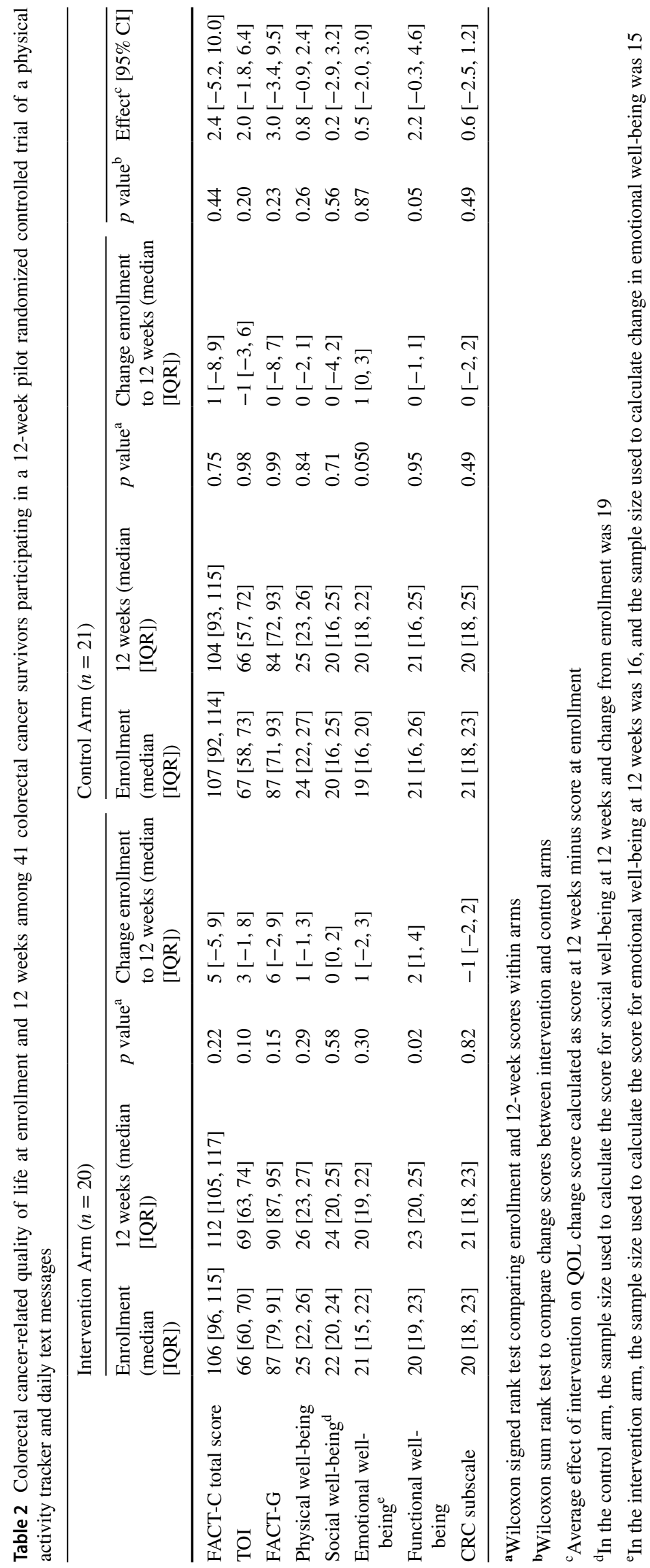




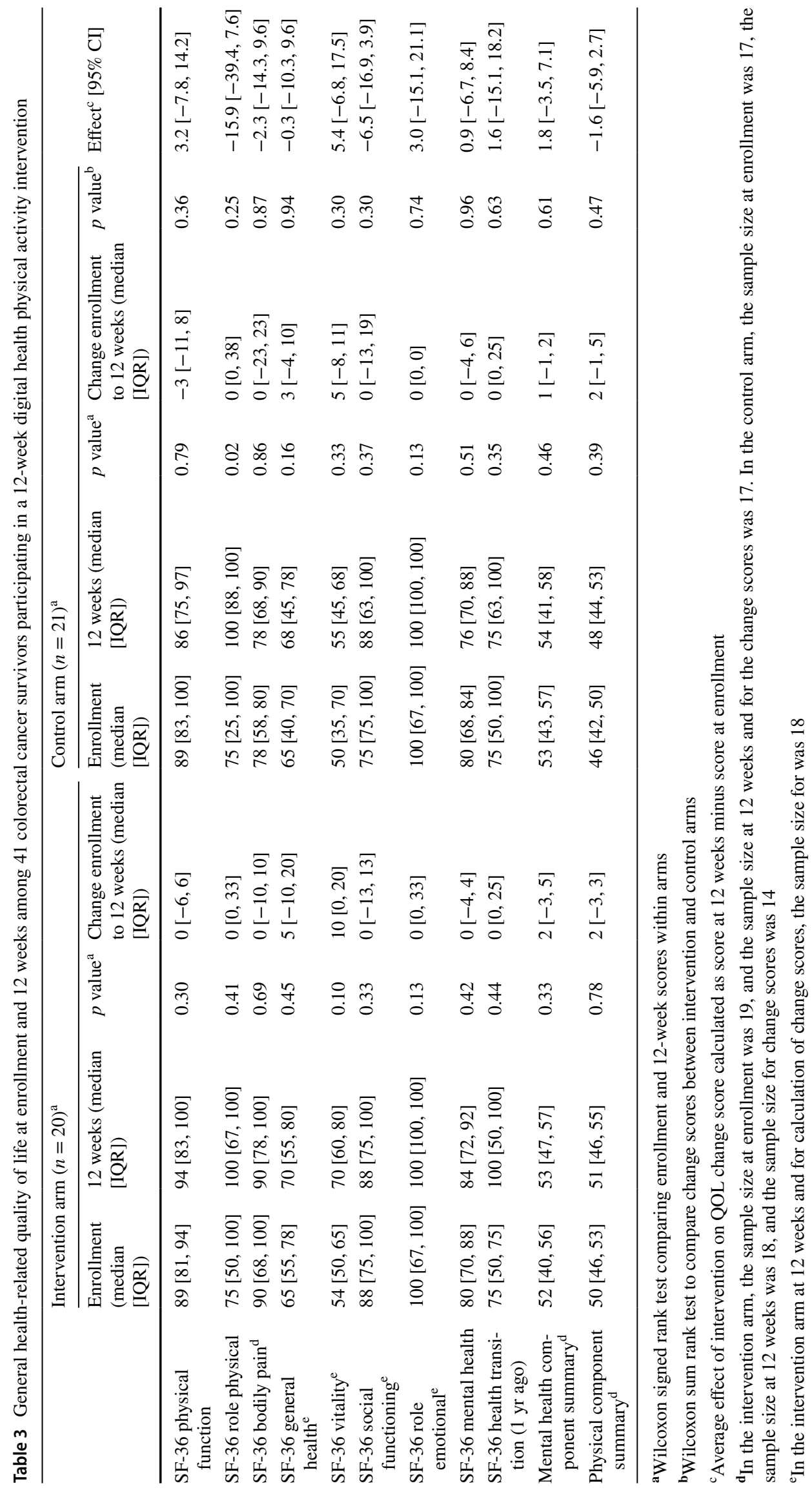


for the US general population (mean $50 \pm 10$ ) [22]. This is consistent with prior studies, which have demonstrated near normalization of global HRQoL scores in CRC survivors 1 year after diagnosis [4]. In addition to enrolling inactive survivors, future studies should also target individuals with low HRQoL at enrollment in order to assess whether these survivors may benefit from a digital health physical activity intervention.

In contrast to the average HRQoL scores observed in our patient population, other studies have reported deficits in specific health domains, such as physical, emotional, and social functioning, years after diagnosis in CRC survivors [2, 5, 7]. While we did not observe a difference in score changes between the intervention and control arm in those domains, we observed a small improvement in functional well-being, a measure of ability to perform normal daily activities, in the intervention arm between enrollment and 12 weeks. Functional well-being differs from physical well-being, which is a measure of a patient's physical symptoms. It is unclear if the increase in the functional well-being subscale by a median of 2 points (IQR, 1,4$)$ represents a clinically significant difference in HRQoL. Minimally important differences in FACT-C scores were found to be 5 to 8 points for the FACT-C total score and 2 to 3 points for the CRC subscale; however, other sub-score minimally important differences have not been established [23]. Overall, while we did not observe a change in overall HRQoL, our pilot study findings suggest physical activity trackers and interactive text messaging may improve functional well-being in CRC survivors.

\section{Limitations}

There were limitations to our study. First, as a pilot study evaluating the feasibility of digital health tools, we were limited by sample size and were not powered to find statistical significance in physical activity or quality of life. Second, a question from the SF-36 survey was unintentionally omitted from the online surveys at all time points, which may have introduced measurement error. However, this error was not differential between arms or time points. Third, our study did not collect data on treatment history or the presence of peripheral neuropathy prior to enrollment, which could impact HRQoL. Finally, our study participants were largely white, college-educated patients, which may limit generalizability to the larger population of CRC survivors. Furthermore, we were limited by the high levels of MVPA and average HRQoL among the participants at enrollment. This may reflect selection bias as patients already engaged in exercise may have been more interested in enrolling in this study. In summary, an important lesson learned was that future studies examining the impact of digital health interventions on HRQoL should target inactive CRC survivors with low HRQoL at enrollment.

\section{Conclusion}

In this pilot study, we observed no change in overall HRQoL in CRC survivors randomized to 12 weeks of physical activity trackers and interactive text messaging or usual care. However, we did observe an improvement in functional wellbeing in the intervention arm. Larger randomized studies are needed to definitively determine if a digital health intervention improves functional well-being among CRC survivors.

Abbreviations BMI: body mass index; CRC: colorectal cancer; FACT-C: Functional Assessment of Cancer Therapy-Colorectal; FACT-G: Functional Assessment of Cancer Therapy-General; HRQoL: health-related quality of life; IQR: interquartile range; MVPA: moderate-to-vigorous physical activity; PF: physical function; RCT: randomized controlled trial; SF-36: Medical Outcomes Study Short Form Survey; TOI: trial outcome index; UCSF: University of California, San Francisco

Supplementary Information The online version contains supplementary material available at https://doi.org/10.1007/s00520-022-06870-5.

Author contribution All authors contributed to the study conception and design. Material preparation, data collection, and analysis were performed by Hilary Chan, Emily Mitchell, Li Zhang, Alan Paciorek, and Erin Van Blarigan. The first draft of the manuscript was written by Hilary Chan, and all authors commented on previous versions of the manuscript. All authors read and approved the final manuscript.

Funding This research was supported by the NIH (K07CA197077, KL2TR000143), Mt. Zion Health Fund, and UCSF Helen Diller Family Comprehensive Cancer Center.

Data availability Data are available upon request.

Code availability Not applicable

\section{Declarations}

Ethics approval Approval was obtained from the ethics committee of the UCSF Institutional Review Board. The procedures used in this study adhere to the tenets of the Declaration of Helsinki.

Consent to participate Informed consent was obtained from all individual participants included in the study.

Consent for publication The authors affirm that human research participants provided informed consent for publication. Any patient identifying information is not included in this manuscript.

Conflict of interest The authors declare no competing interests.

Open Access This article is licensed under a Creative Commons Attribution 4.0 International License, which permits use, sharing, adaptation, distribution and reproduction in any medium or format, as long as you give appropriate credit to the original author(s) and the source, provide a link to the Creative Commons licence, and indicate if changes were made. The images or other third party material in this article are included in the article's Creative Commons licence, unless indicated otherwise in a credit line to the material. If material is not included in the article's Creative Commons licence and your intended use is not 
permitted by statutory regulation or exceeds the permitted use, you will need to obtain permission directly from the copyright holder. To view a copy of this licence, visit http://creativecommons.org/licenses/by/4.0/.

\section{References}

1. Siegel RL, Miller KD, Jemal A (2019) Cancer statistics, 2019. CA: A Cancer J Clinicians 69(1):7-34. https://doi.org/10.3322/ caac. 21551

2. Jansen L, Koch L, Brenner H, Arndt V (2010) Quality of life among long-term ( $\geqslant 5$ years) colorectal cancer survivors - systematic review. Eur J Cancer 46(16):2879-2888. https://doi.org/ 10.1016/j.ejca.2010.06.010

3. Arndt V, Merx H, Stegmaier C, Ziegler H, Brenner H (2004) Quality of life in patients with colorectal cancer 1 year after diagnosis compared with the general population: a population-based study. J Clin Oncol 22(23):4829-4836. https://doi.org/10.1200/ JCO.2004.02.018

4. Kopp I, Bauhofer A, Koller M (2004) Understanding quality of life in patients with colorectal cancer: comparison of data from a randomised controlled trial, a population based cohort study and the norm reference population. Inflamm Res 53(S2):S135. https:// doi.org/10.1007/s00011-004-0361-6

5. Arndt V, Merx H, Stegmaier C, Ziegler H, Brenner H (2006) Restrictions in quality of life in colorectal cancer patients over three years after diagnosis: a population based study. Eur J Cancer 42(12):1848-1857. https://doi.org/10.1016/j.ejca.2006.01.059

6. Ramsey SD, Berry K, Moinpour C, Giedzinska A, Andersen MR (2002) Quality of life in long term survivors of colorectal cancer. Am J Gastroenterol 97(5):1228-1234. https://doi.org/10.1111/j. 1572-0241.2002.05694.x

7. Jansen L, Herrmann A, Stegmaier C, Singer S, Brenner H, Arndt V (2011) Health-related quality of life during the 10 years after diagnosis of colorectal cancer: a population-based study. J Clin Oncol 29(24):3263-3269. https://doi.org/10.1200/JCO.2010.31. 4013

8. Mishra SI, Scherer RW, Geigle PM et al (2012) Exercise interventions on health-related quality of life for cancer survivors. Cochrane Database Systematic Rev 8:CD007566. https://doi.org/ 10.1002/14651858.CD007566.pub2

9. Lynch BM, van Roekel EH, Vallance JK (2016) Physical activity and quality of life after colorectal cancer: overview of evidence and future directions. Expert Rev Qual Life Cancer Care 1(1):923. https://doi.org/10.1080/23809000.2016.1129902

10. van Roekel E, Winkler EA, Bours M et al (2016) Associations of sedentary time and patterns of sedentary time accumulation with health-related quality of life in colorectal cancer survivors. Preventive Med Reports 4:262-269. https://doi.org/10.1016/j.pmedr. 2016.06.022

11. Van Blarigan EL, Chan H, Van Loon K et al (2019) Self-monitoring and reminder text messages to increase physical activity in colorectal cancer survivors (Smart Pace): a pilot randomized controlled trial. BMC Cancer 19(1):218. https://doi.org/10.1186/ s12885-019-5427-5
12. Blanchard CM, Courneya KS, Rodgers WM et al (2002) Determinants of exercise intention and behavior in survivors of breast and prostate cancer: an application of the theory of planned behavior. Cancer Nurs 25(2):88-95. https://doi.org/10.1097/00002820200204000-00002

13. Harris PA, Taylor R, Thielke R, Payne J, Gonzalez N, Conde JG (2009) Research electronic data capture (REDCap) — a metadata-driven methodology and workflow process for providing translational research informatics support. J Biomed Informatics 42(2):377-381. https://doi.org/10.1016/j.jbi.2008.08.010

14. Ward WL, Hahn EA, Mo F, Hernandez L, Tulsky DS, Cella D (1999) Reliability and validity of the functional assessment of cancer therapy-colorectal (FACT-C) quality of life instrument. Qual Life Res 8(3):181-195. https://doi.org/10.1023/a:10088 21826499

15. Webster K, Cella D, Yost K (2003) The functional assessment of chronic illness therapy (FACIT) measurement system: properties, applications, and interpretation. Health Qual life Outcomes 1(1):79. https://doi.org/10.1186/1477-7525-1-79

16. Stewart AL, Hays RD, Ware JE (1988) The MOS short-form general health survey: reliability and validity in a patient population. Medical Care 26(7):724-735. https://doi.org/10.1097/00005650198807000-00007

17. Ware JE, Kosinski M, Bayliss MS, McHorney CA, Rogers WH, Raczek A (1995) Comparison of methods for the scoring and statistical analysis of SF-36 health profile and summary measures: summary of results from the medical outcomes study. Med Care 33(4):AS279

18. Vanhelst J, Mikulovic J, Bui-Xuan G et al (2012) Comparison of two ActiGraph accelerometer generations in the assessment of physical activity in free living conditions. BMC Res Notes 5(1):187. https://doi.org/10.1186/1756-0500-5-187

19. Troiano RP, Berrigan D, Dodd KW, Mâsse LC, Tilert T, McDowell M (2008) Physical activity in the United States measured by accelerometer. Med Sci Sports Exercise 40(1):181. https://doi.org/ 10.1249/mss.0b013e31815a51b3

20. Rosner B (2016) Fundamentals of biostatistics, 8th edn. Cengage Learning, Boston ISBN 9781305268920

21. Rock CL, Doyle C, Demark-Wahnefried W et al (2012) Nutrition and physical activity guidelines for cancer survivors. CA: A Cancer J Clinicians 62(4):242-274. https://doi.org/10.3322/caac. 21142

22. Laucis NC, Bhattacharyya T, Hays RD (2015) Scoring the SF-36 in orthopaedics: a brief guide. J Bone Joint Surg 97(19):16281634. https://doi.org/10.2106/JBJS.0.00030

23. Yost KJ, Cella D, Chawla A et al (2005) Minimally important differences were estimated for the functional assessment of cancer therapy-colorectal (FACT-C) instrument using a combination of distribution- and anchor-based approaches. J Clin Epidemiol 58(12):1241-1251. https://doi.org/10.1016/j.jclinepi.2005.07.008

Publisher's note Springer Nature remains neutral with regard to jurisdictional claims in published maps and institutional affiliations. 